\title{
Interventional Pulmonology and COVID-19: Experience from a Malaysian Tertiary Hospital
}

Nai-Chien Huan, ${ }^{* 1}{ }_{M B B S}$ (Monash), MRCP (UK), Khai Lip Ng, ${ }^{* 1}{ }_{M D}$ (NNSMA), MRCP (UK), Jeat Thong Tang, ${ }^{1} M D(U K M), M R C P(U K)$, Han Nee $\underline{\text { Kua }},{ }^{1} M D(U S M), M R C P(U K)$, Ummi Nadira Daut, ${ }^{2}$ MD (USM), MMed Int Med (UKM), Noorul Afidza

Muhammad, ${ }^{1}$ MD (UKM), MMed Int Med (UKM), Mona Zaria Nasaruddin, ${ }^{1}{ }_{M D}$ (UPM), MMed Int Med (UKM),

Jamalul Azizi Abdul Rahman, ${ }^{1}$ LRCP \& S, MBBChBAO (Ireland), MMed Int Med (UKM)

\begin{abstract}
The ongoing pandemic of COVID-19 has presented multiple challenges to global healthcare services, dictating changes in almost every aspect of daily medical practice. Performing aerosol generating procedures (AGPs) in the field of interventional pulmonology can lead to profound formation of aerosols, leading to a high risk of infection among healthcare workers (HCWs). We share our experiences on performing AGPs in the midst of a COVID-19 pandemic by focusing on changes in AGP practices. In a pandemic, HCWs ought to adapt to the ever-changing situation and use available resources to provide the best possible healthcare to patients, ensure safety of staff, and continue medical education of future pulmonologists.
\end{abstract}

Ann Acad Med Singap 2020;49:1013-7

Keywords: Bronchoscopy, infectious diseases, pulmonary, respiratory medicine

The ongoing pandemic of coronavirus disease 2019 (COVID-19) has led to more than 131,000 confirmed cases and 537 deaths in Malaysia as of 8 January 2021. Malaysia reported its first case of COVID-19 on 25 January 2020. After observing a significant upsurge of cases and recording the first COVID-19 related death on 17 March 2020, Malaysia announced a nationwide Movement Control Order (MCO) on 18 March 2020. ${ }^{1}$

The Department of Pulmonology, Serdang Hospital, Malaysia is a tertiary referral centre for interventional pulmonology (IP) procedures. IP procedures such as flexible and rigid bronchoscopy are generally classified as aerosol generating procedures (AGPs). Performing such procedures can lead to profound formation of aerosols leading to high risks of infection to healthcare workers (HCWs). By May 2020, various international and local recommendations on endoscopy services during the COVID-19 pandemic were already available. ${ }^{2,3}$ The American College of Chest Physicians (CHEST) and the American Association for Bronchology and Interventional Pulmonology (AABIP) guidelines, for example, recommended that in order to maximise protection of patients and $\mathrm{HCWs}$, bronchoscopy should be used sparingly in the evaluation and management of patients with suspected or confirmed COVID-19 infection. ${ }^{2}$ In an area where community transmission of COVID-19 infection is present, bronchoscopy should be deferred for non-urgent indications, and where necessary to perform, HCWs should wear personal protective equipment (PPE) while performing the procedure even on asymptomatic patients. Another article published by Steinfort et al. specifically examined optimal approaches to the selection of IP procedures during the pandemic, which is particularly relevant to centres with IP services. ${ }^{4}$

In this commentary, we share our experiences and insights on performing IP procedures in the COVID-19 pandemic by detailing: (1) changes in AGP practices as a result of COVID-19, (2) steps to ensure safety of HCWs and patients, and (3) impact on training and continuous medical education.

\footnotetext{
${ }^{1}$ Department of Pulmonology, Serdang Hospital, Malaysia

${ }^{2}$ Department of Medicine, Universiti Putra Malaysia, Malaysia

Address for Correspondence: Dr Nai-Chien Huan, Department of Pulmonology, Serdang Hospital, Jln Puchong, 43000 Kajang, Selangor, Malaysia.

Email: khailip.moh@1govuc.gov.my

"Joint first authors
} 
Changes in AGP practices as a result of COVID-19 pandemic. Before the outbreak of COVID-19 in Malaysia, our department had an average of 15 to 20 IP procedures per week, including basic procedures such as flexible bronchoscopy and medical thoracoscopy, to more advanced procedures such as rigid bronchoscopy, electromagnetic navigational bronchoscopy, and airway stenting. We observed a dramatic reduction in the number of procedures after MCO was declared by the federal government of Malaysia (Fig. 1), as all outpatient nonurgent cases were postponed. Postponed cases were subcategorised by the level of urgency. Patients suspected to have lung malignancy or active infection such as tuberculosis were given priorities, while non-urgent cases such as surveillance bronchoscopy post-endobronchial valve insertion for chronic obstructive pulmonary disease, airway stent insertion for benign airway strictures and bronchial thermoplasty, were postponed. Selected cases were discussed during virtual multidisciplinary meetings between pulmonologists, thoracic radiologists, cardiothoracic surgeons and pathologists before final decisions were made. In some instances, board meetings led to decisions against performing certain AGPs, when taking into account the prevailing pandemic situation. An example of a board decision is prioritising percutaneous lung biopsies that carried a lower risk of disease transmission over bronchoscopic biopsies, whenever feasible.

As a tertiary hospital and a major IP centre, we continued to receive and perform IP procedures on emergency cases during the MCO period. During the initial few weeks after the implementation of MCO, AGPs performed were on patients with immediate life-threatening conditions. For example, we performed rigid bronchoscopes for a few patients with malignant central airway occlusion requiring emergency tumour debulking and stenting. For another patient with life-threatening massive haemoptysis leading to asphyxia, rigid bronchoscopes were performed to facilitate clot evacuation.

Besides the postponement of non-urgent cases causing a reduction in the number of AGPs performed, the downward trend could also be due to various reasons, including but not limited to: (1) reduced patient movement and inter-hospital case referrals due to interstate travel restrictions; (2) a general tendency by the public to avoid healthcare facilities due to the fear of contracting a novel virus of uncertain virulence and transmission risk; and (3) concerns among HCWs regarding COVID-19, thereby favouring resource conservation measures.

We recorded a gradual increment in the number of IP cases starting from May 2020. This upward trend coincided with the time when more comprehensive local and international guidelines on performing AGPs during the pandemic were available. In total, we performed 72 IP procedures from 18 March 2020 to 7 July 2020: flexible bronchoscopy (53), pleuroscopy (7), rigid bronchoscopy (10) and indwelling pleural catheter insertions (2). This was in stark contrast to a total of 315 cases performed from February to June 2019: flexible bronchoscopy (214), pleuroscopy (45) and rigid bronchoscopy (56) (Fig. 2). With time, our endoscopy staff became trained on social distancing and infection control measures, especially with regards to proper donning and doffing of PPE. Such measures gave the staff confidence in AGPs during the pandemic.

Steps to ensure safety of HCWs and patients. The need to protect our own staff and patients, especially vulnerable patients such as elderly patients, patients with multiple comorbidities and immunocompromised patients, has led our department to create a simple algorithm describing our institutional guidelines for precautions in patients undergoing IP procedures (Fig. 3).

By April 2020, in line with hospital infection control policy, all patients undergoing surgical procedures including IP procedures were admitted to a temporary pre-procedural ward, a few days before their procedures for close observation and for mandatory pre-procedural COVID-19 nasopharyngeal and/or oropharyngeal polymerase chain reaction (PCR) swabs tests. During patient admission, measures were taken to ensure adequate social distancing and to minimise interpatient contact. For example, beds were placed at least 2 metres apart from each other. Patients were required to wear masks at all times during their stay. Disposable utensils were used while common areas were sanitised frequently. Furthermore, attending staff were required to wear PPE consisting of surgical masks, face shields, gloves and disposable gowns to minimise cross infection. Patients who tested negative for COVID-19 PCR swab tests were transferred out of the pre-procedural ward to their respective wards, while patients who tested positive were promptly sent to COVID-19 isolation wards for further management. These measures were necessary to minimise the risks of disease transmission from patients to $\mathrm{HCWs}$ involved in surgical or endoscopy procedures. From April 2020 until July 2020, we recorded 2 patients from the pre-procedural ward who tested positive for COVID-19. Both patients were otherwise stable.

We advocate pre-procedural COVID-19 PCR swab tests to be done on all patients, especially in areas where community mitigation strategies are in place. It is also vital for endoscopy staff to use full PPE for 


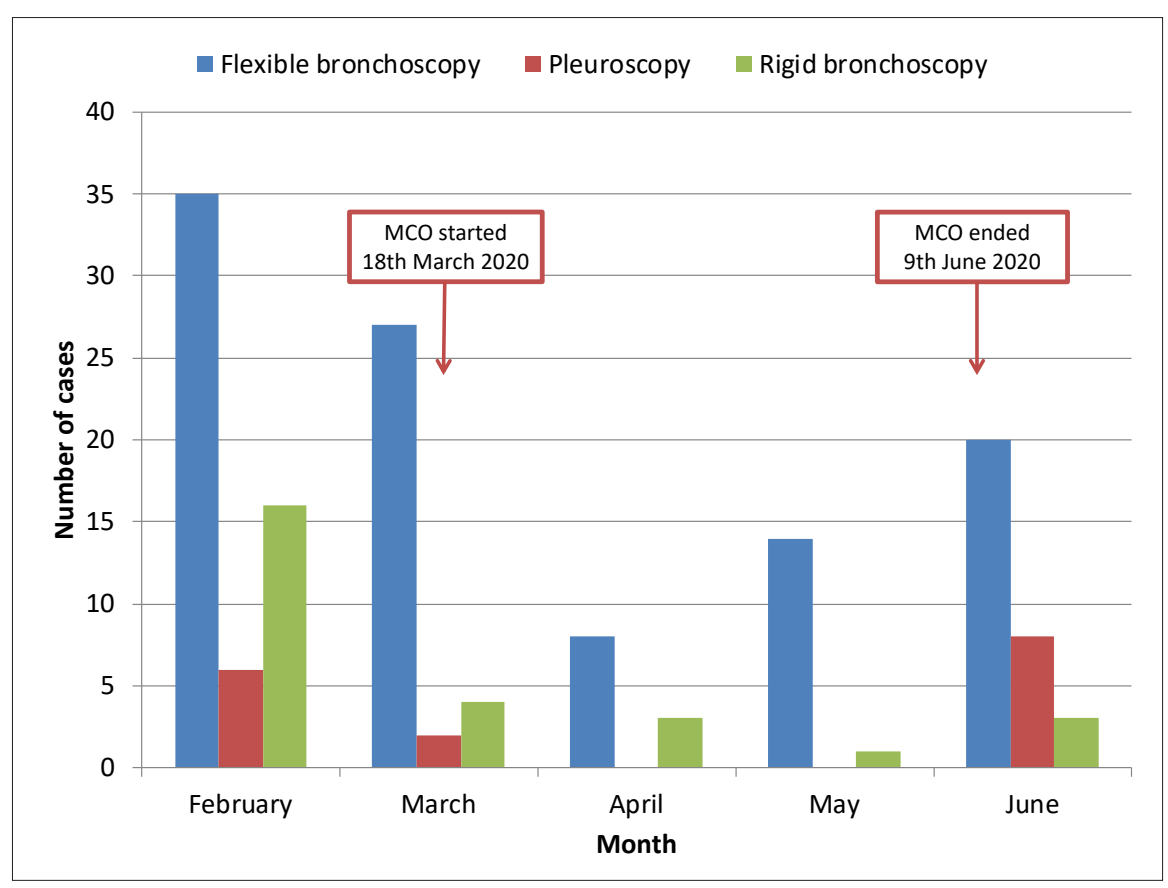

Fig. 1. Number of aerosol-generating procedures at Department of Pulmonology, Serdang Hospital from February 2020 to June 2020. MCO: Movement Control Order.

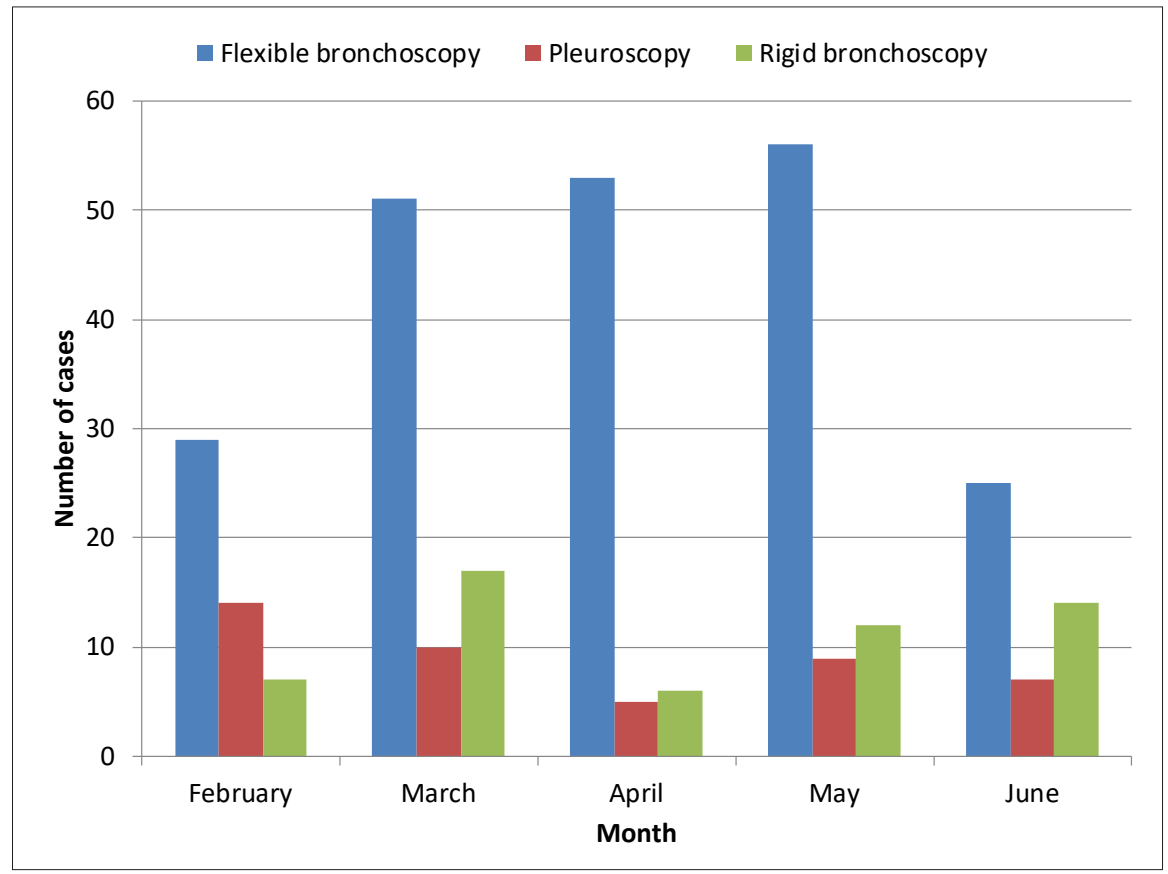

Fig. 2. Number of aerosol-generating procedures at Department of Pulmonology, Serdang Hospital from February 2019 to June 2019. MCO: Movement Control Order.

all procedures. The use of N-95 masks or powered airpurifying respirators should be mandatory when dealing with patients with suspected or confirmed COVID-19 infection, regardless of whether they are symptomatic or not. Such a practice is in tandem with other disciplines, e.g. dentistry, where there is a high risk of COVID-19 transmission from AGPs. ${ }^{5}$ We strongly discourage the practice of 2-tier PPE usage between different teams, e.g. the endoscopy team wearing N-95 masks, while supporting anaesthesia members wear surgical masks. 


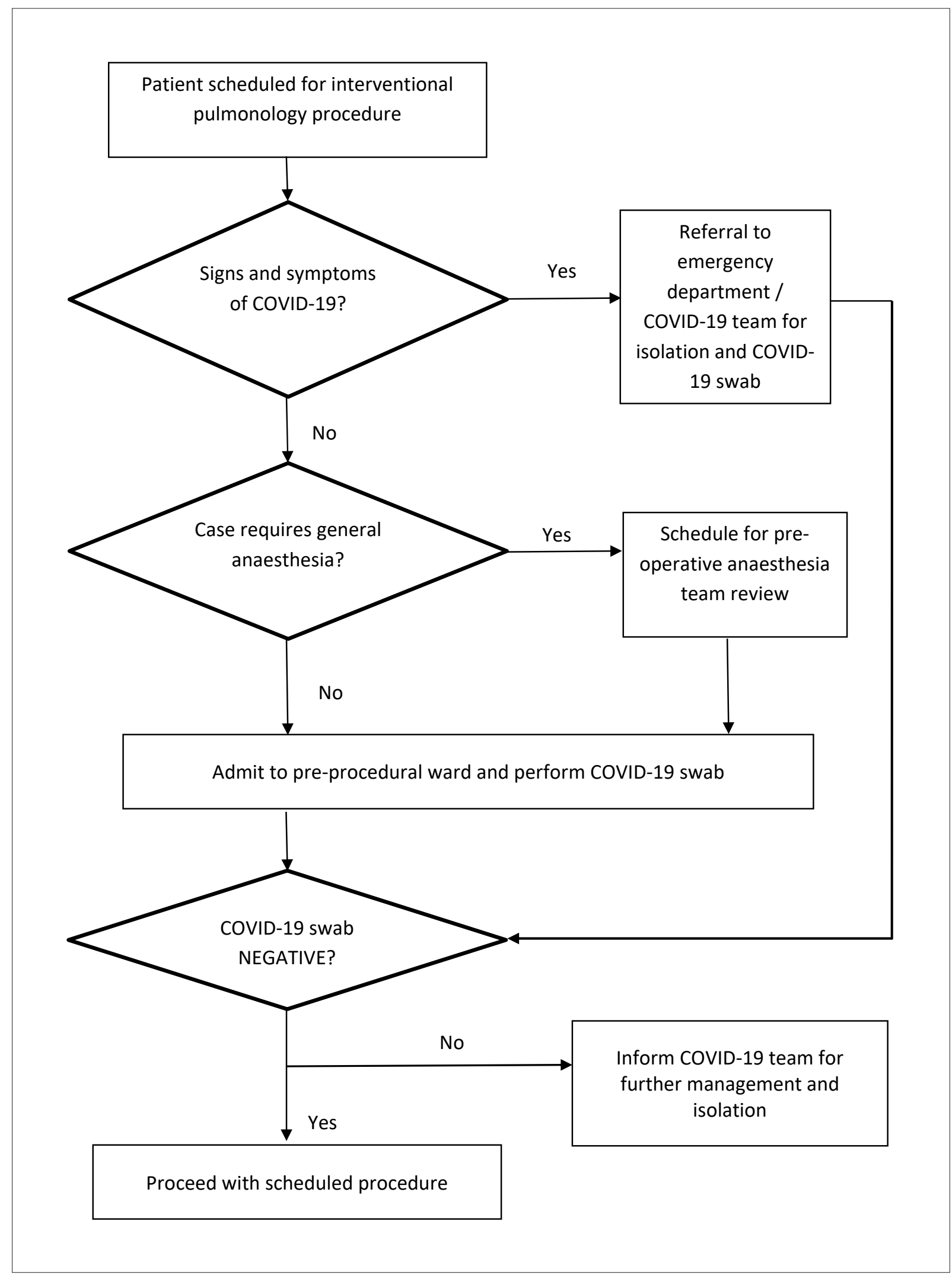

Fig. 3. Algorithm describing institutional precautions for patients undergoing interventional pulmonology-related procedures. 
All symptomatic endoscopy staff are required to undergo COVID-19 PCR swab tests; they are relieved of their duties until their test results are negative.

Impact on training and continuous medical education. There are ongoing concerns on whether the reduction in procedures will affect the training schedule and proficiency of pulmonology and anaesthesia trainees. Most endoscopy suites including ours have implemented measures to reduce the number of personnel during AGPs. This approach may mean that junior trainees are deterred from observing or directly participating in AGPs. Trainees may fear that their endoscopy skills will gradually diminish due to less time spent in endoscopy suites. Conversely, HCWs have justifiable needs for work safety, especially for those with elderly, young or sick family members at home. During such times, it is important to manage the anxieties of colleagues by assuring them that there are good evidences for the overall efficacy of PPE. ${ }^{6}$ Training programmes can still be conducted during a pandemic through the increased use of online learning such as instructional videos or laboratory simulations, to avoid unnecessary crowding of procedure rooms. When face-to-face teaching sessions were cancelled at the beginning of the MCO, we resorted to online educational platforms. Online learning platforms allow rapid sharing of large amounts of information between local and foreign participants and speakers, in a user-friendly, easily accessible and flexible manner.

However, like a double-edged sword, the pandemic does present new opportunities. Current restrictions on performing AGPs encourage clinicians to rely on, cherish and re-explore the basics of physiology and diagnostic modalities, focusing on non-invasive tests before considering AGPs. Moreover, it encourages the entire respiratory fraternity to engage, research and explore innovative diagnostic methods focusing on new frontiers such as liquid biopsy or reliable biomarkers. We hope the pandemic serves to accelerate and catalyse such innovations to reduce the reliance on invasive procedures in the future.

In conclusion, the unprecedented COVID-19 pandemic has long-term effects, leading to changes in almost every aspect of healthcare. HCWs need to adapt to the ever-changing situation and use their current available resources to provide the best possible healthcare to patients, ensure safety of staff, and continue medical education of future pulmonologists.

\section{REFERENCES}

1. Ministry of Health Malaysia. COVID-19 Malaysia. Available at: http:// covid-19.moh.gov.my/. Accessed on 10 July 2020.

2. Wahidi MM, Shojaee S, Lamb CR, et al. The use of bronchoscopy during the coronavirus disease 2019 pandemic: CHEST/AABIP guideline and expert panel report. Chest 2020;158:1268-81.

3. The Malaysian Thoracic Society recommendations on endoscopy services during COVID-19 pandemic, 25 March 2020. Available at: http://mts.org.my/download/MTS_Recommendations_on_Endoscopy_ Services_COVID-19_Pandemic.pdf. Accessed on 8 July 2020.

4. Steinfort DP, Herth FJ, Irving LB, et al. Safe performance of diagnostic bronchoscopy/EBUS during the SARS-CoV-2 pandemic. Respirology 2020;25:703-8.

5. Shi $\mathrm{AH}$, Guo $\mathrm{W}$, Chng $\mathrm{CK}$, et al. Precautions when providing dental care during coronavirus disease 2019 (COVID-19) pandemic. Ann Acad Med Singap 2020;49:312-19.

6. Wang X, Pan Z, Cheng Z. Association between 2019-nCov transmission and N95 respirator use. J Hosp Infect 2020;105:104-5. 